\title{
Highly efficient blue light-emitting diodes with tunable wavelength using vertically graded bandgap quasi-2D perovskite films
}

\section{Zhiyong Fan ( $\nabla$ eezfan@ust.hk)}

Hong Kong University of Science and Technology https://orcid.org/0000-0002-5397-0129

\section{Lei Shu}

Hong Kong University of Science and Technology

Qianpeng Zhang

Hong Kong University of Science and Technology

\section{Swapnadeep Poddar}

Hong Kong University of Science and Technology

\section{Daquan Zhang}

The Hong Kong University of Science and Technology

\section{Yu Fu}

Hong Kong University of Science and Technology

\section{Bryan Cao}

Hong Kong University of Science and Technology

\section{Yucheng Ding}

Hong Kong University of Science and Technology

\section{Article}

Keywords: light-emitting diodes, perovskite materials, PeLEDs

Posted Date: September 29th, 2021

DOI: https://doi.org/10.21203/rs.3.rs-926924/v1

License: (c) (1) This work is licensed under a Creative Commons Attribution 4.0 International License.

Read Full License 


\title{
Highly efficient blue light-emitting diodes with tunable wavelength using vertically graded bandgap quasi-2D perovskite films
}

Lei Shu ${ }^{l}$, Qianpeng Zhang ${ }^{1}$, Swapnadeep Poddar ${ }^{1}$, Daquan Zhang ${ }^{l}, Y u \mathrm{Fu}^{l}$, Bryan Cao $^{l}$, Yucheng Ding ${ }^{1}$, and Zhiyong Fan ${ }^{1,2 *}$

${ }^{1}$ Department of Electronic and Computer Engineering, The Hong Kong University of Science and Technology, Clear Water Bay, Kowloon, Hong Kong SAR, China

${ }^{2}$ Guangdong-Hong Kong-Macao Intelligent Micro-Nano Optoelectronic Technology Joint Laboratory, The Hong Kong University of Science and Technology, Clear Water Bay, Kowloon, Hong Kong SAR, China.

* Corresponding to: eezfan@ust.hk

\begin{abstract}
Metal halide perovskite materials have emerged as a unique class of solution process compatible semiconductors and alluring candidates for high-performance optoelectronic applications ${ }^{1,2,3}$, especially light-emitting diodes (LEDs), owing to high quantum efficiency, facile color tunability, narrow emission line widths, as well as costeffectiveness $^{4,5,6}$. Despite of the great successes on green and red perovskite LEDs (PeLEDs), the advancement of external quantum efficiency (EQE) of blue PeLEDs still lags far behind those of green and red PeLEDs ${ }^{7,8}$. Here, we demonstrate color-tunable blue PeLEDs devices with high EQE of $16.1 \%$ and $10 \%$ for emission wavelengths of $472 \mathrm{~nm}$ and $461 \mathrm{~nm}$, respectively. The efficient tunable wavelength electroluminescence (EL) and
\end{abstract}


high EQE originate from the optimization of the recombination zone position to reach the charge injection balance in the vertically graded bandgap quasi-2D perovskite materials. Under the synergetic effect of lead chloride $\left(\mathrm{PbCl}_{2}\right)$ doping and propane-1,3-diammonium $\left(\mathrm{PDABr}_{2}\right)$ incorporation, the vertically graded bandgap perovskite materials can be prepared by the self-regulation of the reduced-dimensional perovskite during the annealing process. Our work here has significantly elevated the performance of the current blue PeLEDs. It opens up a novel avenue to fabricate high-performance blue PeLEDs that can match up the performance of the green and red PeLEDs for future lighting and display applications.

\section{Introduction}

Blue light-emitting diodes (LEDs) are critical components in solid-state lighting and full-color displays. Nevertheless, it is particularly challenging to develop blue perovskite LEDs (PeLEDs) with an emission wavelength shorter than $475 \mathrm{~nm}^{2}$. Recently, PeLEDs with emission wavelength from near-infrared to green have achieved EQEs above $20 \%^{7,9,10,11,12}$. However, the performance of blue PeLEDs still lags far behind because blue emissive perovskite materials usually are plagued by deficient stability and quantum efficiency ${ }^{13}$. Previous literature have reported two primary strategies for developing blue PeLEDs, namely, perovskite compositional tuning and quantum confinement (QC) engineering ${ }^{13}$. By tuning the ratio of halides, mostly $\mathrm{Cl}$ and $\mathrm{Br}$, in the perovskite nanocrystals, emission from deep blue to green can be realized. For example, blue ( $477 \mathrm{~nm})$ and deep-blue (467 $\mathrm{nm}$ ) LEDs based on the mixed halide three-dimensional (3D) perovskites with modest $\mathrm{EQE}$ values of $11 \%$ and $5.5 \%$ were realized by using a vaporassisted crystallization technique, generating the 3D perovskites with compositional 
homogeneity ${ }^{14}$. However, the local compositional heterogeneity of mixed halides in lead octahedrons produces ionic defects, leading to ion migration and phase segregation issues, and consequently resulting in the redshift of the emission spectrum under electrical bias or light illumination ${ }^{15}$. In parallel, reduced dimensional (e.g., quasi-2D) perovskite materials are being explored to realize blue emission by inserting organic ammonium ligand as a spacer in between 2D inorganic octahedron lattice layers to convert the 3D bulk perovskite into a $2 \mathrm{D}$ composite material ${ }^{16,17}$. In this scenario, each $2 \mathrm{D}$ lattice layer provides a $\mathrm{QC}$ effect for carriers, thus leading to widened energy bandgap $\left(E_{g}\right)$. And the overall $E_{g}$ of a low-dimensional domain is determined by the number of constituent lattice layers $(n)$ stacking up without ligands. For example, for the $\mathrm{CsPbBr}_{3}$-based quasi-2D perovskites, in principle the $E_{g}$ can be tuned from about $2.6 \mathrm{eV}(n=4, \lambda=477 \mathrm{~nm})$ to $2.7 \mathrm{eV}(n=3, \lambda=460$ $\mathrm{nm}), 2.9 \mathrm{eV}(n=2, \lambda=427 \mathrm{~nm})$, and $3.1 \mathrm{eV}(n=1, \lambda=400 \mathrm{~nm})$ respectively, by reducing the number of the octahedron layers ${ }^{2,18}$. For previously reported quasi-2D perovskite films, an inhomogeneous energy landscape consists of multiple $n$ value phases ${ }^{19}$. Accordingly, by cascade energy transfer process, the energy is channeled across the inhomogeneous energy landscape, guiding carriers to the lowest bandgap $n$ phase which improves the emission efficiency. However, because carries are guided to the lowest bandgap $n$ phase, even there are larger bandgap $n$ phases in quasi-2D perovskite films, thus only long wavelength emission such as sky-blue emission can be achieved ${ }^{18,20}$. Therefore, it is still challenging to achieve pure blue or deep blue emissions based on quasi-2D perovskite materials. To address this challenge, in this report, we demonstrate a highly efficient blue PeLED based on a unique vertically graded bandgap perovskite material $\left(\mathrm{PDACs}_{\mathrm{n}-1} \mathrm{~Pb}_{\mathrm{n}}\left(\mathrm{Br}_{0.77} \mathrm{Cl}_{0.37}\right)_{3 \mathrm{n}+1}\right)$. Herein, the short length organic ligand $\mathrm{PDABr}_{2}$ is used to improve the carrier transfer 
between various $n$ phases to improve the carrier injection efficiency. We dope the $\mathrm{PbCl}_{2}$ into the perovskite precursor solution to prolong the distribution of $\mathrm{PDA}^{2+}$ cations to rearrange the distribution of various $n$ phases in the films thus forming a vertically graded bandgap structure. In addition, the $\mathrm{PbCl}_{2}$ can stabilize the precursor phase and slow down the crystallization process of perovskite ${ }^{21}$. Under the synergetic effect of $\mathrm{PbCl}_{2}$ and $\mathrm{PDA}^{2+}$ cations, the vertically graded bandgap perovskite materials can be obtained by the selfregulation of the reduced-dimensional perovskite during the annealing process. Due to the unique vertically graded bandgap $n$ phase in quasi-2D perovskite films, the lateral bandgap variation at the recombination zone is small enough to hinder the cascade energy transfer, avoiding the redshift of the EL peak, and therefore resulting in the pure blue emission.

Intriguingly, it was discovered that by tuning the thicknesses of hole transporting layer (HTL) poly(3,4-ethylenedioxythiophene) polystyrene sulfonate (PEDOT: PSS) and electron transporting layer (ETL) 1,3,5-benzinetriyl)tris(1-phenyl-1H-benzimidazole (TPBi), the charge injection balance can be modulated and the recombination zone position can be rationally shifted to the specific region of the quasi-2D perovskite film. As a result, to the best of our knowledge, the tunable color of pure blue (472 $\mathrm{nm}$ ) and deep blue (461 nm) ELs are achieved with the record-high EQE values of $16.1 \%$ and $10 \%$, respectively. This work demonstrates the alluring potency to use well-engineered quasi-2D perovskites for high-performance PeLEDs and reveals the importance of controlling the distribution of low-dimensional structures as well as recombination zone position inside the material.

\section{Results and Discussion}

Cl doping effect on the film properties. It is well known that all inorganic $\mathrm{CsPbCl}_{3}$ perovskite with large $E_{g}$ at the deep blue region is an ideal candidate for the pure blue 
PeLEDs $^{22}$. However, the poor solubility of $\mathrm{CsPbl}_{3}$ in dimethyl sulfoxide (DMSO) hinders the formation of 3D bulk $\mathrm{CsPbCl}_{3}$ film for the pure blue PeLEDs. Meanwhile, inorganic $\mathrm{CsPbBr}_{3}$ perovskite materials have excellent solubility in $\mathrm{DMSO}$. Bulk $\mathrm{CsPbBr}_{3}$ usually has green emission and reducing the dimension to 2D leads to $\mathrm{QC}$ effect, yielding pure/deep blue emissions ${ }^{2,23}$. It was reported recently that stable low-dimensional $\mathrm{CsPbBr}_{3}$ domains with aromatic poly ammonium bromide [1,4-bis (aminomethyl)benzene bromide $(\mathrm{P}-\mathrm{PDABr} 2)]$ spacer could achieve a deep-blue emission with a modest EQE of $2.6 \%{ }^{2}$. In our work here, propane-1,3-diammonium bromide $\left(\mathrm{PDABr}_{2}\right)$, cesium bromide $(\mathrm{CsBr})$, lead chloride $\left(\mathrm{PbCl}_{2}\right)$, and lead bromide $\left(\mathrm{PbBr}_{2}\right)$ with molar ratios of 0.17: 0.31: 0.18: 0.12 are used to form $\mathrm{PDACs}_{n-1} \mathrm{~Pb}_{\mathrm{n}}\left(\mathrm{Br}_{0.55} \mathrm{Cl}_{0.37}\right)_{3 \mathrm{n}+1}$. The $\mathrm{PDABr}_{2}$ is used to fabricate reduceddimensional perovskite.

A compact and pinhole-free perovskite film is vital for high device performance. Therefore, the scanning electron microscope (SEM) was used to characterize the morphology of the perovskite thin films with various $\mathrm{PbCl}_{2}$ to $\mathrm{PbBr}_{2}$ molar ratios. Fig. 1a shows that the pure $\mathrm{PDACS}_{n-1} \mathrm{~Pb}_{n} \mathrm{Br}_{3 n+1}$ film has many pinholes and uniform grain size distribution. However, after introducing $\mathrm{PbCl}_{2}$, the morphology is significantly improved, as shown in Fig. S1. Fig. 1b shows the pinhole-free perovskite film with uniform crystal size when the molar ratio of $\mathrm{PbCl}_{2}$ over $\mathrm{PbBr}_{2}$ is optimized to 0.6: 0.4. In addition, when the molar ratio of $\mathrm{PbCl}_{2}$ to $\mathrm{PbBr}_{2}$ is increased to 1.0: 0.0, the pinholes in the perovskite film re-appear (in Fig. S1).

Moreover, the atomic concentration depth analysis by Kratos X-ray photoelectron spectrometer (Axis Ultra DLD by Kratos) on perovskite films with 0.0:1.0 and 0.6:0.4 $\mathrm{PbCl}_{2}$ to $\mathrm{PbBr}_{2}$ molar ratios are shown in Fig. 1c and Fig. 1d, respectively. For perovskite 
film without $\mathrm{Cl}$ doping (0.0:1.0 molar ratio), the concentration of $\mathrm{N}$ element (from the organic ligand $\mathrm{PDABr}_{2}$ ) has a small distribution depth, and it rapidly decreases, which indicates the presence of quasi-2D component comprising $\mathrm{PDABr}_{2}$ specifically close to the upper surface of the film, as shown in Fig. 1c. After introducing $\mathrm{PbCl}_{2}$ into perovskite materials, the distribution depth of $\mathrm{N}$ element increased, as shown in Fig. 1d. Although the $\mathrm{N}$ element concentration also experiences a fast decay, but it possesses a long tail, as shown in Fig. 1d. Therefore, introducing $\mathrm{PbCl}_{2}$ not only improves the morphology of perovskite film but also increases the distribution depth of quasi-2D perovskites in the film. Other atomic concentration depth analysis results with different molar ratios of $\mathrm{PbCl}_{2}$ to $\mathrm{PbBr}_{2}$ are shown in Fig. S2. It is known that the formation and distribution of low-dimensional perovskite domains in perovskite film are determined by the incorporated organic spacer cations ${ }^{24,25}$. As shown in Fig. 1e, after introducing $\mathrm{PbCl}_{2}$, the distribution depth of $\mathrm{PDA}^{2+}$ cations in perovskite film is extended. There are more organic ligands in the upper layer region, leading to a smaller $n$ number and stronger QC. Towards the bottom of the film, less ligands render a larger $n$ number, generating more 3D bulk-like properties.

Fig. 1f shows the photoluminescence (PL) spectra of perovskite film with different $\mathrm{PbCl}_{2}: \mathrm{PbBr}_{2}$ molar ratios. The $\mathrm{PL}$ peak position of pure $\mathrm{Br}$ perovskite $\left(\mathrm{PDACs}_{\mathrm{n}-1} \mathrm{~Pb}_{\mathrm{n}} \mathrm{Br}_{3 \mathrm{n}+1}\right)$ is $491 \mathrm{~nm}$, which indicates the formation of quasi-2D perovskites with QC effect. With the increase of the molar ratio of $\mathrm{PbCl}_{2}$ in the precursor solution, the PL peak position of the perovskite film shifts from sky-blue $(491 \mathrm{~nm})$ to deep blue $(450 \mathrm{~nm})$ region. The UV-vis absorption spectra of the perovskite film with different $\mathrm{PbCl}_{2}$ concentrations are shown in Fig. S3. All samples present two excitonic absorption peaks and the shorter wavelength one can be assigned to low-dimensional domains. In addition, with the increase of the 
concentration of $\mathrm{PbCl}_{2}$, both absorption peaks are blue-shifted, which means the $E_{g}$ is increased. As shown in Fig. 1g, the photoluminescence quantum yields (PLQYs) of $\mathrm{PDACs}_{\mathrm{n}-1} \mathrm{~Pb}_{\mathrm{n}} \mathrm{Br}_{3 \mathrm{n}+1}$ is $57.1 \%$. Improving perovskite material quality such as minimizing bulk, surface, and interface defects to reduce the non-radiative recombination rate is a common approach to increase $\mathrm{PLQY}{ }^{26}$. As the molar ratio of $\mathrm{PbCl}_{2}$ to $\mathrm{PbBr}_{2}$ increases to 0.2:0.8 and 0.4:0.6, the PLQYs increase to $67.37 \%$ and $77.3 \%$, respectively. However, the PLQY slightly decreases to $76.9 \%$ as the molar ratio of $\mathrm{PbCl}_{2}$ to $\mathrm{PbBr}_{2}$ increases to $0.6: 0.4$. This PLQY change in sync with the perovskite film quality observed with SEM for varying $\mathrm{Cl} / \mathrm{Br}$ ratios. A pin-hole-free film genuinely has fewer defects. Then the PLQY starts to plummet as the $\mathrm{PbCl}_{2}$ molar ratio is further increased. Furthermore, the time-resolved photoluminescence (TRPL) spectra in Fig. S4 show that Cl-doped perovskite films have a radiative lifetime ( $\tau=4.55 \mathrm{~ns})$ longer than that of the pure $\mathrm{Br}$ perovskite film $(\tau=1.92 \mathrm{~ns})$. For halide perovskite, the longer lifetime of photoluminescence transition is a direct evidence of reduced defects ${ }^{9,27,28}$. Therefore, the defects of perovskite material are reduced after introducing a certain concentration of $\mathrm{PbCl}_{2}$.

Formation of vertically graded bandgaps in quasi-2D perovskite film. The X-ray diffraction (XRD) measurement is performed to study the crystal structural property of the perovskite films. According to Fig. 2a, for the pure Br PDACs ${ }_{n-1} \mathrm{~Pb}_{n} \mathrm{Br}_{3 n+1}$ thin film, the diffraction peaks marked with a black vertical solid line at $14.6^{\circ}, 23.7^{\circ}, 29.4^{\circ}$, and $36^{\circ}$ are the same as the diffraction patterns of the 3D perovskite film. The diffraction peaks marked with the red dashed line at $10.3^{\circ}$ are attributed to the quasi-2D perovskite with $n=1^{28}$. The diffraction peak marked with a blue dash line at $11.8^{\circ}$ is ascribed to $n=2$ or the quasi-2D perovskite mixing with other phases such as $n=3^{28,29}$. After introducing $\mathrm{PbCl}_{2}$ into $\mathrm{PDACs}_{\mathrm{n}}$ 
${ }_{1} \mathrm{~Pb}_{\mathrm{n}} \mathrm{Br}_{3 \mathrm{n}+1}$ perovskite, only the $n=1$ quasi-2D perovskite diffraction peak at $10.3^{\circ}$ can be observed, the higher $n$ quasi-2D perovskite diffraction peak at $11.8^{\circ}$ disappears. Therefore, the introduction of $\mathrm{PbCl}_{2}$ affects the distribution of the low-dimensional quasi-2D perovskite in the film.

To further explore the distribution of quasi-2D perovskites in the film, a systematic investigation on the PL spectra of perovskite thin film at different depths is conducted. Fig. 2b presents the normalized PL spectra of the perovskite film with $\mathrm{PbCl}_{2}: \mathrm{PbBr}_{2}=0.6: 0.4$ molar ratio on ITO/ PEDOT: PSS substrate after argon ion milling to remove the surface material in a layer-by-layer fashion (details can be found in Method section). In the subsequent experiments, all devices are fabricated with the perovskite film with $\mathrm{PbCl}_{2}$ : $\mathrm{PbBr}_{2}=0.6: 0.4$ molar ratio. By this method, the perovskite film can be thinned down layer by layer with high controllability using argon ion milling process and thus the optical properties of the perovskite film at different depths can be investigated. Specifically, we acquired PL spectra by exciting the perovskite film from the upper surface using excitation wavelength of $350 \mathrm{~nm}$, with the results shown in Fig. 2c. It can be seen that before the ion milling process, the PL peak is centered at $465 \mathrm{~nm}\left(E_{g}=2.62 \mathrm{eV}\right)$. When the ion milling time increases, the PL peak red-shifts and eventually reaches $491 \mathrm{~nm}$ with $15 \mathrm{~min}$ ion milling time. These results indicate that after spin coating perovskite on glass/PEDOT:PSS substrate, the upper layer of the film has small $n$ number while the lower layer close to the PEDOT:PSS interface has larger $n$ number. The trend of gradual red-shift when moving toward the PEDOT:PSS/glass substrate suggests that a vertically graded bandgap structure has been formed wihin the perovskite film. Intriguingly, we discover that the PL spectra appear to have long wavelength emission tail for the film before ion milling and after short 
ion milling time ( $<12 \mathrm{~min}$ ), as shown in Fig. S5. Especially for the PL acquired after 8 mins ion milling (Fig. S6), there is a shoulder peak appearing at $491 \mathrm{~nm}$ with the main emission peak positioned at $472 \mathrm{~nm}$. This indicates the long wavelength PL emission from the bottom of the film with large $n$ number quasi-2D perovskite can also show up when the film is thinned down to a proper thickness. To further verify this, we have excited PL of the perovskite film with 8 min ion milling from the glass substrate side. In this case, the bottom of the perovskite film is exposed to excitation source. As shown in Fig. 2d, interestingly, the $493 \mathrm{~nm}$ emission peak is much more obvious now. This confirms that the large $n$ number quasi-2D perovskite PL emission is from the bottom of the perovskite film. Meanwhile, the $472 \mathrm{~nm}$ emission peak still has significantly higher intensity than the 493 $\mathrm{nm}$ peak. This can be rationalized by considering the charge transfer into PEDOT:PSS and the resulting PL quenching effect for the large $n$ number quasi-2D perovskite which is adjacent to PEDOT:PSS.

The above optical property study has proven that after spin coating perovskite on glass/PEDOT:PSS substrate, the upper layer of the film has small $n$ numbered quasi-2D perovskite while the bottom layer close to the PEDOT:PSS interface has large $n$ numbered quasi-2D perovskite. To further verify the existence of different $n$ numbers and their distribution trend, high resolution transmission electron microscopy (HRTEM) from the cross section of the perovskite film is obtained, as shown in Fig. 2e. In the upper layer, HRTEM shows the smallest lattice spacing of $3.04 \AA$ A, corresponding to the (040) plane of the perovskite and a corresponding peak at $29.6^{\circ}$ in the XRD pattern shown in Fig. 2a. Therefore $n=1$ is observed here ${ }^{30}$. For the middle layer, the lattice fringes show a periodicity of $6.08 \AA$ which is two times of $3.04 \AA$, thus indicating $n=2$ in this region. And 
at the lower part of the film, the lattice fringes with a periodicity of $12.16 \AA$ can be seen, revealing existence of quasi-2D perovskite with $n=4$ in this region. These results clearly verify that we have the vertically graded bandgap perovskite material with different $n$ numbered layers.

Color tunable blue emission from PeLED devices. Since our above PL results have shown that the upper layer of the perovskite film has $E_{g}=2.62 \mathrm{eV}$ (465 nm emission) and the lower layer of the perovskite film possesses $E_{g}=2.48 \mathrm{eV}$ (491 nm emission), in principle, deep blue to sky blue color EL emission can be achieved via controlling the carrier recombination zone from the upper layer to lower layer of the quasi-2D perovskite film with graded bandgap. Intriguingly, this has been successfully implemented in our work via systematically tuning the thicknesses of ETL and HTL, and the results are shown and discussed below. Fig. 3a shows the schematic of the EL device architecture with indiumdoped tin oxide (ITO)/PEDOT:PSS/perovskite/TPBi/lithium fluoride (LiF)/aluminum (Al) from the bottom to top. The flat-band energy level diagram is shown in Fig. 3b. The valance band position of the perovskite film is determined from the ultraviolet photoelectron spectroscopy (UPS) (Fig. S7), and the $E_{v}$ of perovskite film with $\mathrm{PbCl}_{2}$ : $\mathrm{PbBr}_{2}=0.6: 0.4$ molar ratio is $-5.97 \mathrm{eV}$. In addition, the cutoff energy of the perovskite film is gradually increased with the increase of the $\mathrm{PbCl}_{2}$ concentration. We discovered that the recombination zone position in the perovskite film can be rationally controlled by tuning the thicknesses of the HTL or ETL. As shown in the schematic diagram Fig. 3c, the position of the recombination zone descends gradually from the TPBi/perovskite interface to the perovskite/PEDOT:PSS interface when increasing PEDOT:PSS thickness or decreasing the TPBi thickness. According to this scenario, carriers recombine at different depths, as 
shown in Fig. 3d, generating different EL wavelengths. To verify this hypothesis, we performed a systematic experiment as follows.

Firstly, the TPBi layer thickness is fixed at $50 \mathrm{~nm}$ to study the impact of PEDOT:PSS thickness on the device performance. The normalized device EL spectra for different PEDOT: PSS thicknesses varying from $38 \mathrm{~nm}$ to $48 \mathrm{~nm}$ are shown in Fig. S8. The thickness of PEDOT:PSS is controlled by the spin-coating condition and is characterized by ellipsometer. As the holes are injected from the PEDOT:PSS side, increasing its thickness slows down hole injection and consequently the electron-hole recombination zone moves toward the PEDOT: PSS/perovskite interface. It is worth noting that this recombination zone tuning approach has also been reported previously ${ }^{20}$. As PEDOT:PSS thickness is increased, the EL peak is red-shifted from $469 \mathrm{~nm}$ to $484 \mathrm{~nm}$ correspondingly with a monotonic trend, as shown in Fig. 3e. This confirms that our perovskite film has a vertically graded bandgap structure inside. As shown in Fig. S9, the EQEs of the devices with various thicknesses of PEDOT:PSS are achieved. The peak EQE of $8.6 \%$ is obtained for the device with $42 \mathrm{~nm}$ PEDOT:PSS, which can be attributed to the charge injection balance with the optimal HTL thickness. The corresponding $J-L-V$ curves of the devices with different PEDOT:PSS thicknesses are given in Fig. S10.

Likewise, it is also observed that the recombination zone position can be tuned by changing the TPBi thickness. In this case, the PEDOT:PSS thickness is fixed at $48 \mathrm{~nm}$ and TPBi thickness is systematically increased from $14 \mathrm{~nm}$ to $50 \mathrm{~nm}$. The thickness of TPBi can be well controlled in the evaporation process (Method). As shown in Fig. 3f, an overall blue shift of EL peak position (from $471 \mathrm{~nm}$ to $461 \mathrm{~nm}$ ) is observed when increasing the TPBi thickness from $14 \mathrm{~nm}$ to $50 \mathrm{~nm}$ (in Fig. S11). This can be rationalized by considering 
the fact that thickening TPBi slows down electron injection thus moving the electron-hole recombination zone towards the perovskite/TPBi interface. Due to the existence of low- $n$ dimensional domains (larger bandgap) near the perovskite/TPBi interface, a shorter EL emission wavelength is obtained. The EQEs of devices with various TPBi thicknesses are also achieved, as shown in Fig. S12. The corresponding $J-V-L$ curves of the devices with different TPBi thicknesses are shown in Fig. S13. Notably, the EQE of the device with 50 $\mathrm{nm}$ TPBi reaches $\sim 10 \%$ with a deep blue $(461 \mathrm{~nm})$ emission, which greatly exceeds that of previously reported deep blue PeLEDs. While tuning the thicknesses of TPBi and PEDOT:PSS, various EL peak positions are obtained due to the fact that the recombination zone position can be modulated to different depths in the quasi-2D perovskite film.

The above results and discussions confirm that a unique vertically graded bandgap quasi$2 \mathrm{D}$ perovskite is formed in the film. The distribution of reduced-dimensional quasi-2D perovskite in film is controlled by the intercalating cation, stoichiometry of the precursor, and the deposition process (such as annealing) ${ }^{31,32}$. The doping of $\mathrm{PbCl}_{2}$ can prolong the distribution depth of $\mathrm{PDA}^{2+}$ cations in perovskite film, which determines the distribution of $n$ values phase. Moreover, the presence of $\mathrm{PbCl}_{2}$ can stabilize the precursor phase and slow down the crystallization process of the perovskite ${ }^{21}$. On the other hand, the $\mathrm{DMSO}^{33}$ and low temperature annealing process are used to slow down the growth rate of perovskite crystal to allow the self-regulation of the reduced-dimensional perovskite to form the vertically graded bandgap quasi-2D structure.

High quantum efficiency pure blue PeLED. The wavelength-tunable blue PeLEDs with high EQE can be achieved based on the vertically graded bandgap quasi-2D perovskite by optimizing the recombination zone position. And by accurately controlling the thicknesses 
of HTL and ETL, the charge injection balance can be obtained. According to the result in Fig. S9 and S12, when the recombination zone is positioned is at zone A (Fig. 4a), the highest $\mathrm{EQE}$ with best charge injection balance is obtained, and the EL emission wavelength is between $465 \mathrm{~nm}$ and $477 \mathrm{~nm}$. Therefore, after further optimization of the thicknesses of TPBi and PEDOT:PSS, the EQE of 14 devices are shown in Fig. 4b. The champion device with EQE of $16.1 \%$ is achieved with optimal TPBi $(\sim 33 \mathrm{~nm})$ and PEDOT: PSS ( 42 nm) thicknesses. The cross-sectional SEM image of the champion device is shown in Fig. S14. The EL result of this device structure is shown in Fig. 4c, demonstrating the symmetric pure blue EL spectrum with a peak position located at about $472 \mathrm{~nm}$ and a narrow full-width at half-maximum (FWHM) of $25 \mathrm{~nm}$. The corresponding Commission International de l'Eclairage (CIE) color coordinate is (0.1303, 0.1124) (Fig. S15), demonstrating high color purity of blue PeLEDs. Fig. 4d shows the current density $(J)$ luminance $(L)$-voltage $(V)$ curves of the champion blue PeLEDs with a maximum brightness of $188 \mathrm{~cd} \mathrm{~m}^{-2}$. The device's peak EQE is $16.1 \%$, as shown in Fig. 4e. To the best of our best knowledge, this is the highest EQE for pure blue PeLEDs reported so far (Fig. 4f). Fig. 4f shows the benchmark of our device performance with the existing literature. It can be seen that the uniquely graded bandgap quasi-2D perovskite film structure has significantly elevated the record EQEs of PeLEDs with pure blue and deep blue emission. Moreover, T50 of the device with an initial brightness of $100 \mathrm{~cd} \mathrm{~m}^{-2}$ is measured as 11.3 min, as shown in Fig. S16. There are a few possible reasons that limit the lifetime of blue perovskite LEDs. For example, the mixed halide perovskite has a phase segregation issue, which causes device degradation to affect the lifetime of devices.

\section{Conclusion}


In this work, vertically graded bandgap quasi-2D perovskite films are formed by a synergetic effect of $\mathrm{PDA}^{2+}$ ions incorporation and $\mathrm{Cl}$ doping in perovskite crystallization process. Such films have been used as the core emissive layer for high efficiency PeLEDs. Intriguingly, by controlling the thicknesses of hole and electron injection layers to change the position of the recombination zone, the blue emission wavelength of the devices based on the same perovskite materials can be tuned from $461 \mathrm{~nm}$ to $484 \mathrm{~nm}$. Within this range, both $461 \mathrm{~nm}$ wavelength deep blue emission device and $472 \mathrm{~nm}$ wavelength pure blue emission device demonstrate record high EQE of $10 \%$ and $16.1 \%$, respectively. Overall, the unique graded bandgap quasi-2D perovskite thin film structure developed here opens up a new vista to achieve deep and pure blue PeLEDs with high efficiencies. And the charge injection tunability demonstrated here can potentially be harnessed for tunable color PeLED in broad applications for lighting and display applications in the future. 


\section{Materials and Methods}

\section{Materials}

Poly(3,4-ethylenedioxythiophene) polystyrene sulfonate (PEDOT: PSS) (AI 4083) was purchased from Ossila. All the other chemicals, $\mathrm{CsBr}, \mathrm{PbBr}_{2}, \mathrm{PbCl}_{2}, \mathrm{PDABr}_{2}, \mathrm{TPBi}, \mathrm{LiF}$, dimethyl sulfoxide (DMSO) were purchased from Sigma-Aldrich and used as received withour further purification.

\section{Perovskite precursor solution preparation}

The perovskite precursor solution was prepared by dissolving $\mathrm{PDABr}_{2}, \mathrm{CsBr}, \mathrm{PbBr}_{2}$, and $\mathrm{PbCl}_{2}$ in DMSO, with the molar ratio of $\mathrm{PDABr}_{2}$ : $\mathrm{CsBr}$ : $\left(\mathrm{PbBr}_{2}+\mathrm{PbCl}_{2}\right)$ equal to 0.169: 0.308: 0.308. Different concentrations of $\mathrm{PbCl}_{2}$ (molar ratio of $\mathrm{PbBr}_{2}: \mathrm{PbCl}_{2}$ equal to 1.0: $0.0,0.8: 0.2,0.6: 0.4,0.4: 0.6$, and $0.0: 1.0)$ were introduced into the perovskite precursor solutions. The perovskite precursor solutions were heated and stirred at $75^{\circ} \mathrm{C}$ for $2 \mathrm{~h}$ and then the solution was subjected to continuous overnight stirring. All the above experiments pertaining to the solution preparation was done in ambient air condition.

\section{Blue PeLEDs fabrication}

The patterned ITO-coated glass substrates were sequentially sonicated with deionized water, isopropyl alcohol, acetone, and then blown dry by nitrogen. ITO glass substrates were treated with oxygen plasma with a power of $100 \mathrm{~W}$ for $15 \mathrm{~min}$. Then the PEDOT: PSS (AI 4083) hole transporting layer was prepared by spin-coating PEDOT: PSS aqueous solution onto the ITO substrate at 3,600 rpm for $1 \mathrm{~min}$ and then baked at $155^{\circ} \mathrm{C}$ for $20 \mathrm{~min}$ in ambient air. By tuning the spin-coating rates, the thickness of PEDOT: PSS can be adjusted. Then the perovskite precursor solution was spin-coated onto the PEDOT: PSS 
film at $4200 \mathrm{rpm}$ for $1 \mathrm{~min}$ and then annealed at $85^{\circ} \mathrm{C}$ for $10 \mathrm{~min}$. The electron transport layer of TPBi was deposited on the perovskite film by using thermal evaporation with a $0.2 \mathrm{~nm} \mathrm{~s}^{-1}$ rate under a vacuum of $<4 \times 10^{-4} \mathrm{~Pa}$, and different thicknesses of TPBi were deposited. Finally, $1 \mathrm{~nm} \mathrm{LiF}$ and $100 \mathrm{~nm} \mathrm{Al}$ were deposited using the same evaporation system. The overlapping area of ITO and Al electrodes is the device area $\left(\sim 2.25 \mathrm{~mm}^{2}\right)$.

\section{Characterization}

Field-emission scanning electron microscopy (JEOL JSM-7800F) was used to characterize the top-view morphology of the perovskite film with different $\mathrm{PbCl}_{2}$ concentrations. The PL, PLQY, and TRPL were performed with Edinburgh Instruments FLS920P. For the ion milling etching process, the accelerating voltage is $120 \mathrm{~V}$, and the title angle between the perovskite films and the argon ion beam is about $70^{\circ} \sim 80^{\circ}$ during the etching process. The chamber pressure is about $4 \times 10^{-4} \mathrm{~Pa}$ and Ar gas flow is $4 \sim 12 \mathrm{sccm}$. TEM model is JEMARF200F (JEOL) and FIB Model is FEI HELIOS G4 UX with a dual beam FIB/FESEM system. The TEM sample was prepared by cross sectional cutting, lifted off and attached with the help of dual beam FIB. The final thinning voltage used was $2 \mathrm{kV}$ and the lamella thickness was less than $100 \mathrm{~nm}$. The atom concentration was measured by Kratos X-ray photoelectron spectrometer (Axis Ultra DLD by Kratos). The sputtering conditions: Ar, $4 \mathrm{kV}, 3 \times 3 \mathrm{~mm}$ raster, $140 \mu \mathrm{A}$ extractor current. The performance of blue PeLEDs was measured by using a source-measurement-unit Keithley 2400 and a spectrometer (Ocean Optics, Flame-S-VIS-NIR-ES) ${ }^{45}$. The XRD spectra of the perovskite films were measured on a Rigaku Smart Lab $(\lambda=1.54 \AA)$. 


\section{Acknowledgements}

This work was financially supported by Shenzhen Fundamental Research Program (Project No. JCYJ20170818114107730), Hong Kong Research Grant Council (General Research Fund Project No. 16214619, 16237816, 16309018), Hong Kong Innovation Technology Fund (GHP/014/19SZ), HKUST Fund of Nanhai (Grant No. FSNH18FYTRI01), Guangdong-Hong Kong-Macao Intelligent Micro-Nano Optoelectronic Technology Joint Laboratory (project no. 2020B1212030010), and Foshan Innovative and Entrepreneurial Research Team Program (2018IT100031). The authors also acknowledge the support from the Center for 1D/2D Quantum Materials and the State Key Laboratory of Advanced Displays and Optoelectronics Technologies at HKUST. We thank Y. Zhu for assistance with the SEM measurement, B. Ren \& Z. Ma \& C. L. J. Chan \& C. Wang \& X. Qiu for assistance with the data analysis, M. Qin for assistance with the XRD measurements.

\section{Author contributions}

Z. Fan and L. Shu conceived the idea and designed the experiments. Z. Fan supervised the work. L. Shu, Q. Zhang, and Z. Fan wrote the manuscript and did the data analysis. L. Shu and S. Poddar carried out the TEM measurement and FIB cutting. L. Shu, Q. Zhang, D. Zhang, Y. Fu, and B. Cao carried out the device fabrication and characterizations. Y. Ding, participated in data analysis and paper revision. All authors contribute to the paper discussion and agree to the results.

The authors declare no conflict of interests. 


\section{References}

1. Jeon, N. J. et al. Compositional engineering of perovskite materials for highperformance solar cells. Nature 517, 476-480 (2015).

2. Yuan, S. et al. Optimization of Low-Dimensional Components of Quasi-2D Perovskite Films for Deep-Blue Light-Emitting Diodes. Adv. Mater. 31, 1-9 (2019).

3. Gu, L. et al. A biomimetic eye with a hemispherical perovskite nanowire array retina. Nature 581, 278-282 (2020).

4. Zhang, X. et al. Hybrid Perovskite Light-Emitting Diodes Based on Perovskite Nanocrystals with Organic-Inorganic Mixed Cations. Adv. Mater. 29, 1-7 (2017).

5. Jiang, Y. et al. Spectra stable blue perovskite light-emitting diodes. Nat. Commun. 10, 1-9 (2019).

6. Wang, H. et al. Trifluoroacetate induced small-grained $\mathrm{CsPbBr} 3$ perovskite films result in efficient and stable light-emitting devices. Nat. Commun. 10, (2019).

7. Kim, Y. H. et al. Comprehensive defect suppression in perovskite nanocrystals for high-efficiency light-emitting diodes. Nat. Photonics 15, 148-155 (2021).

8. Hassan, Y. et al. Ligand-engineered bandgap stability in mixed-halide perovskite LEDs. Nature 591, 72-77 (2021).

9. Lin, K. et al. Perovskite light-emitting diodes with external quantum efficiency exceeding 20 per cent. Nature 562, 245-248 (2018).

10. Cao, Y. et al. Perovskite light-emitting diodes based on spontaneously formed submicrometre-scale structures. Nature 562, 249-253 (2018).

11. Chiba, T. et al. Anion-exchange red perovskite quantum dots with ammonium iodine salts for highly efficient light-emitting devices. Nat. Photonics 12, 681-687 (2018).

12. $\mathrm{Xu}, \mathrm{W}$. et al. Rational molecular passivation for high-performance perovskite lightemitting diodes. Nat. Photonics 13, 418-424 (2019).

13. Chu, Z. et al. Large cation ethylammonium incorporated perovskite for efficient and spectra stable blue light-emitting diodes. Nat. Commun. 11, 1-8 (2020).

14. Karlsson, M. et al. Mixed halide perovskites for spectrally stable and high-efficiency blue light-emitting diodes. Nat. Commun. 12, 1-10 (2021).

15. Zhang, H. et al. Phase segregation due to ion migration in all-inorganic mixed-halide perovskite nanocrystals. Nat. Commun. 10, 1-8 (2019).

16. Kumar, S. et al. Efficient Blue Electroluminescence Using Quantum-Confined TwoDimensional Perovskites. ACS Nano 10, 9720-9729 (2016).

17. Vashishtha, P. et al. High Efficiency Blue and Green Light-Emitting Diodes Using Ruddlesden-Popper Inorganic Mixed Halide Perovskites with Butylammonium 
Interlayers. Chem. Mater. 31, 83-89 (2019).

18. Xing, J. et al. Color-stable highly luminescent sky-blue perovskite light-emitting diodes. Nat. Commun. 9, 1-8 (2018).

19. Yuan, M. et al. Perovskite energy funnels for efficient light-emitting diodes. Nat. Nanotechnol. 11, 872-877 (2016).

20. Li, Z. et al. Modulation of recombination zone position for quasi-two-dimensional blue perovskite light-emitting diodes with efficiency exceeding 5\%. Nat. Commun. 10, 1-10 (2019).

21. Stone, K. H. et al. Transformation from crystalline precursor to perovskite in $\mathrm{PbCl} 2-$ derived MAPbI3. Nat. Commun. 9, (2018).

22. Protesescu, L. et al. Nanocrystals of Cesium Lead Halide Perovskites $\left(\mathrm{CsPbX}_{3}, \mathrm{X}=\right.$ $\mathrm{Cl}, \mathrm{Br}$, and I): Novel Optoelectronic Materials Showing Bright Emission with Wide Color Gamut. Nano Lett. 15, 3692-3696 (2015).

23. Zheng, X. et al. Chlorine Vacancy Passivation in Mixed Halide Perovskite Quantum Dots by Organic Pseudohalides Enables Efficient Rec. 2020 Blue Light-Emitting Diodes. ACS Energy Lett. 5, 793-798 (2020).

24. Wu, C. et al. Alternative Type Two-Dimensional-Three-Dimensional Lead Halide Perovskite with Inorganic Sodium Ions as a Spacer for High-Performance LightEmitting Diodes. ACS Nano 13, 1645-1654 (2019).

25. Hu, J. et al. Synthetic control over orientational degeneracy of spacer cations enhances solar cell efficiency in two-dimensional perovskites. Nat. Commun. 10, 111 (2019).

26. Zhang, D. et al. Increasing Photoluminescence Quantum Yield by Nanophotonic Design of Quantum-Confined Halide Perovskite Nanowire Arrays. Nano Lett. 19, 2850-2857 (2019).

27. Yang, W. S. et al. Iodide management in formamidinium-lead-halide-based perovskite layers for efficient solar cells. Science (80-. ). 356, 1376-1379 (2017).

28. Yang, X. et al. Efficient green light-emitting diodes based on quasi-two-dimensional composition and phase engineered perovskite with surface passivation. Nat. Commun. 9, 2-9 (2018).

29. Yang, G. et al. Improved current efficiency of quasi-2D multi-cation perovskite light-emitting diodes: The effect of Cs and K. Nanoscale 12, 1571-1579 (2020).

30. Chen, Z. et al. Stable $\mathrm{Sn} / \mathrm{Pb}$-Based Perovskite Solar Cells with a Coherent 2D/3D Interface. iScience 9, 337-346 (2018).

31. Cheng, L. et al. Multiple-Quantum-Well Perovskites for High-Performance LightEmitting Diodes. Adv. Mater. 32, 1-9 (2020).

32. Quintero-Bermudez, R. et al. Compositional and orientational control in metal halide perovskites of reduced dimensionality. Nat. Mater. 17, 900-907 (2018). 
33. Zhou, M., Fei, C., Sarmiento, J. S. \& Wang, H. Manipulating the Phase Distributions and Carrier Transfers in Hybrid Quasi-Two-Dimensional Perovskite Films. Sol. RRL 3, 1-9 (2019).

34. Congreve, D. N. et al. Tunable Light-Emitting Diodes Utilizing Quantum-Confined Layered Perovskite Emitters. ACS Photonics 4, 476-481 (2017).

35. Deng, W. et al. Organometal Halide Perovskite Quantum Dot Light-Emitting Diodes. Adv. Funct. Mater. 26, 4797-4802 (2016).

36. Chiba, T. et al. Blue Perovskite Nanocrystal Light-Emitting Devices via the Ligand Exchange with Adamantane Diamine. Adv. Opt. Mater. 8, 1-7 (2020).

37. Zhang, B. Bin et al. General Mild Reaction Creates Highly Luminescent OrganicLigand-Lacking Halide Perovskite Nanocrystals for Efficient Light-Emitting Diodes. J. Am. Chem. Soc. 141, 15423-15432 (2019).

38. Yao, J. et al. Calcium-tributylphosphine oxide passivation enables the efficiency of pure-blue perovskite light-emitting diode up to $3.3 \%$. Sci. Bull. 65, 1150-1153 (2020).

39. Tan, Z. et al. Spectrally Stable Ultra-Pure Blue Perovskite Light-Emitting Diodes Boosted by Square-Wave Alternating Voltage. Adv. Opt. Mater. 8, 1-8 (2020).

40. Liu, Y. et al. Efficient blue light-emitting diodes based on quantum-confined bromide perovskite nanostructures. Nat. Photonics 13, 760-764 (2019).

41. Dong, Y. et al. Bipolar-shell resurfacing for blue LEDs based on strongly confined perovskite quantum dots. Nat. Nanotechnol. 15, 668-674 (2020).

42. Wang, F. et al. High Performance Quasi-2D Perovskite Sky-Blue Light-Emitting Diodes Using a Dual-Ligand Strategy. Small 16, 2-9 (2020).

43. Wang, Q. et al. Efficient sky-blue perovskite light-emitting diodes via photoluminescence enhancement. Nat. Commun. 10, (2019).

44. Pang, P. et al. Rearranging Low-Dimensional Phase Distribution of Quasi-2D Perovskites for Efficient Sky-Blue Perovskite Light-Emitting Diodes. ACS Nano 14, 11420-11430 (2020).

45. Zhang, Q. et al. Efficient metal halide perovskite light-emitting diodes with significantly improved light extraction on nanophotonic substrates. Nat. Commun. 10, 1-9 (2019). 


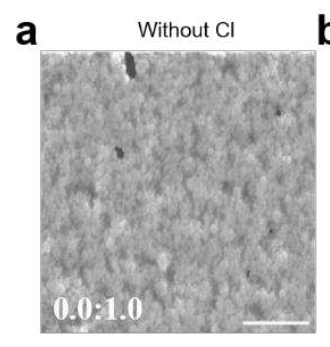

e

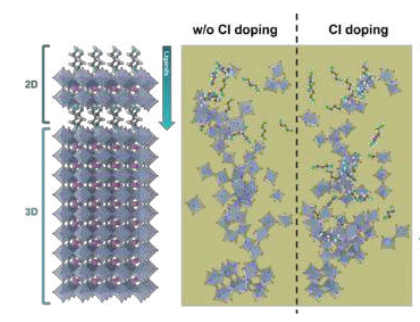

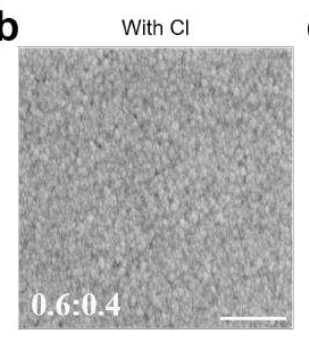

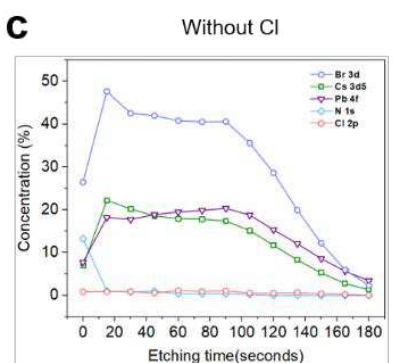

f
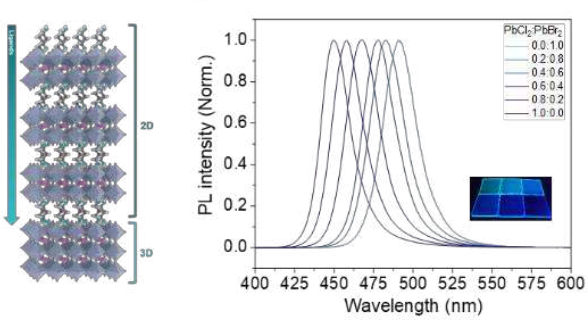

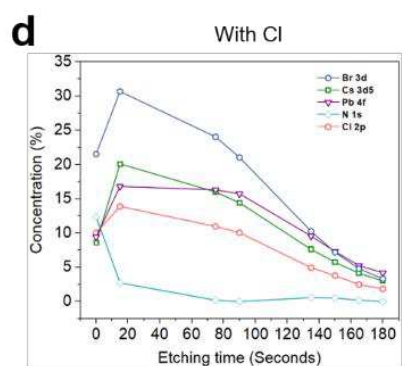

g

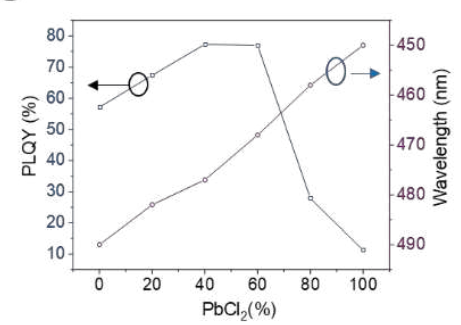

Fig. 1. Cl doping effect on the film properties. The top-view SEM images of perovskite film ( $\left.\mathrm{PDACs}_{n-1} \mathrm{~Pb}_{\mathrm{n}} \mathrm{Br}_{3 \mathrm{n}+1}\right)$ a, without and $\mathrm{b}$, with $\mathrm{Cl}$ doping, the scale bars are $1 \mu \mathrm{m}$. The atomic concentration depth analysis of perovskite film (PDACs $\left.\mathrm{P}_{n-1} \mathrm{~Pb}_{n} \mathrm{Br}_{3 n+1}\right) \mathrm{c}$, without and $\mathrm{d}$, with $\mathrm{Cl}$ doping. $\mathrm{Cl} / \mathrm{Br}$ ratio in $\mathrm{d}$ is $0.6 / 0.4$. e, the schematic of perovskite films with and without $\mathrm{Cl}$ doping. The distribution depth of organic ligands can be prolonged after doping Cl. $\mathrm{f}$, the normalized PL spectra of the perovskite film with different $\mathrm{PbCl}_{2}: \mathrm{PbBr}_{2}$ molar ratios. Inset: the photograph of the perovskite film with different $\mathrm{PbCl}_{2}: \mathrm{PbBr}_{2}$ molar ratios under $365 \mathrm{~nm}$ UV light illumination, the size of the photographed sample is $2 \times 2 \mathrm{~cm}^{2} . \mathrm{g}$, the PLQYs of perovskite film with different $\mathrm{PbCl}_{2}$ : $\mathrm{PbBr}_{2}$ molar ratios and the corresponding PL peak positions. 

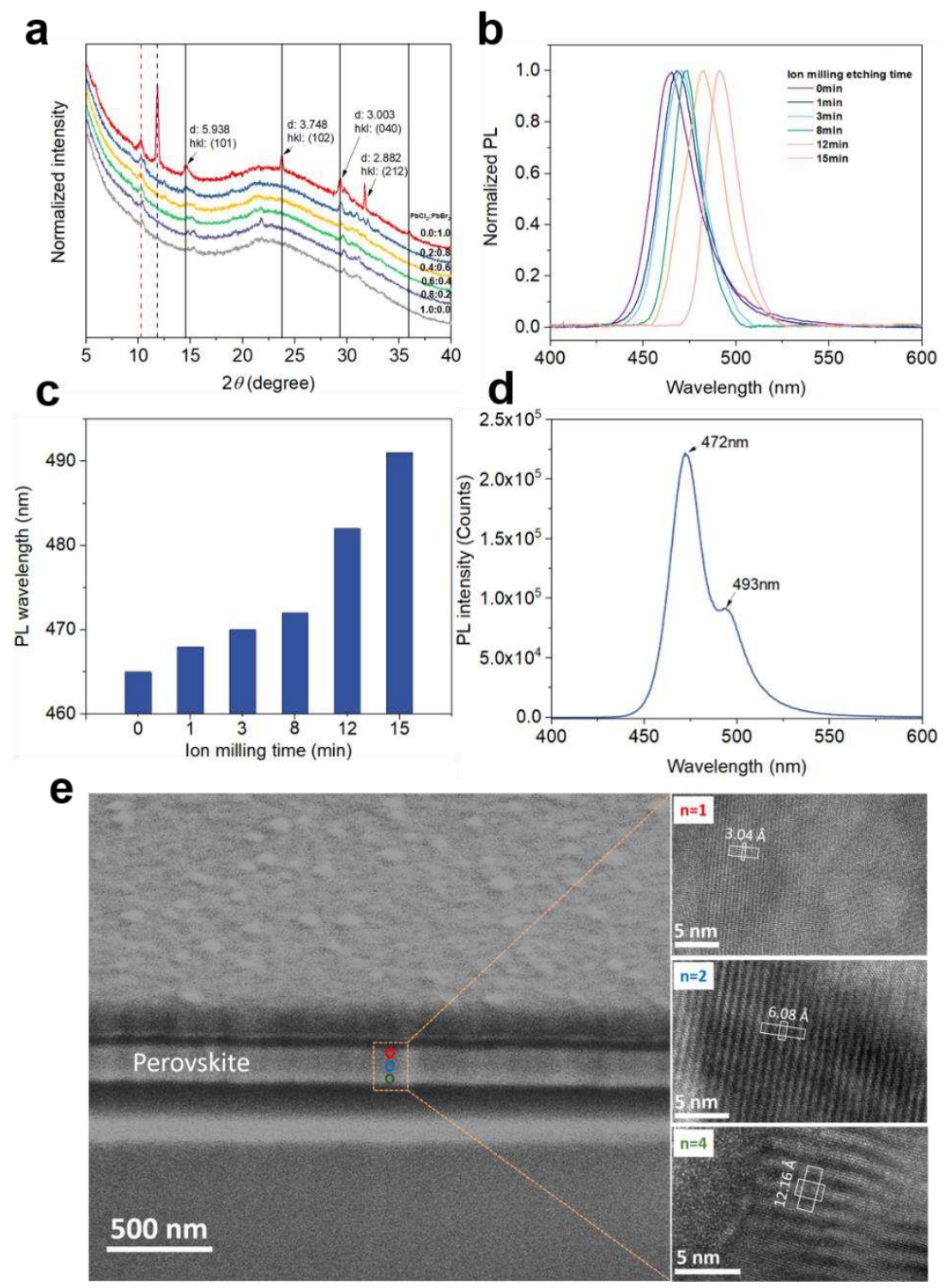

Fig. 2. Formation of the vertically graded perovskite film. a, X-ray diffraction (XRD) patterns of the perovskite film with different $\mathrm{PbCl}_{2}$ to $\mathrm{PbBr}_{2}$ molar ratios. b, the normalized PL spectra of the perovskite film with $\mathrm{PbCl}_{2}: \mathrm{PbBr}_{2}=0.6$ : 0.4 ratio with varying ion milling time (sample excited from airside). c, The EL peak position of the perovskite with varying ion milling time. $d$, The PL spectra of the perovskite film excited from the glass side. e. The TEM images of different $n$ value phases at different depths from the cross section part of the perovskite film. 

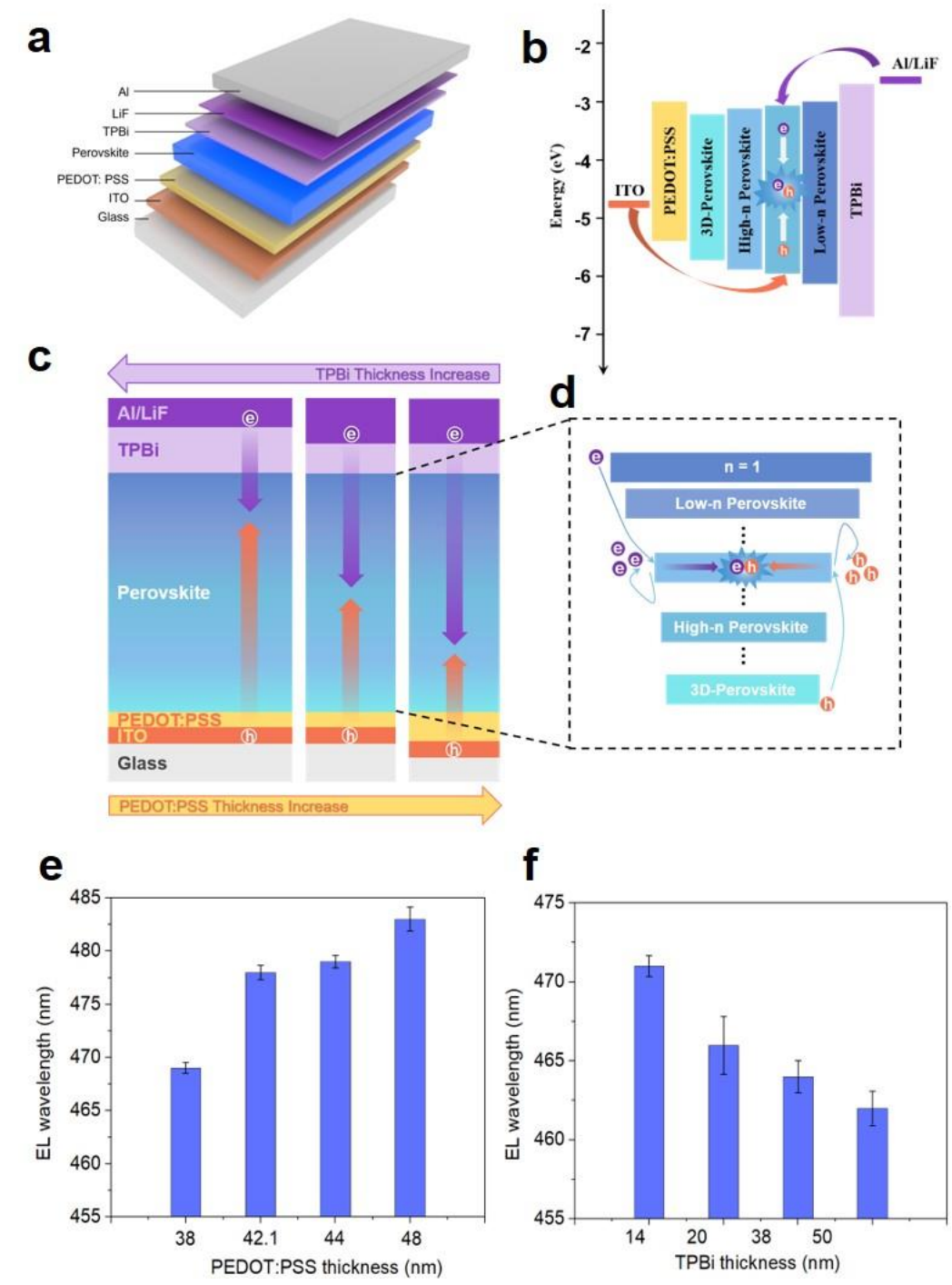

Fig. 3. The modulation of recombination zone position within the perovskite emitting layer. a, the device architecture. b, schematic of flat-band energy level diagram of blue PeLEDs. c, the schematic diagram showing that through tuning the thicknesses of TPBi and PEDOT: PSS, the recombination zone position can be vertically shifted in emitting perovskite layer, therefore varying emission wavelengths can be obtained. $d$. The schematic diagram of the vertically graded bandgap quasi-2D perovskites. e, EL wavelengths of blue PeLEDs with varying PEDOT: PSS thicknesses. f, EL wavelengths of blue PeLEDs with varying TPBi thicknesses. 
a
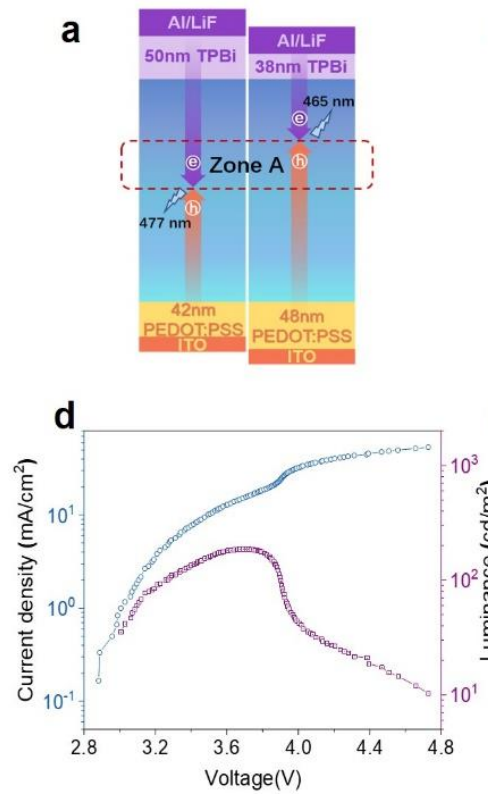

b
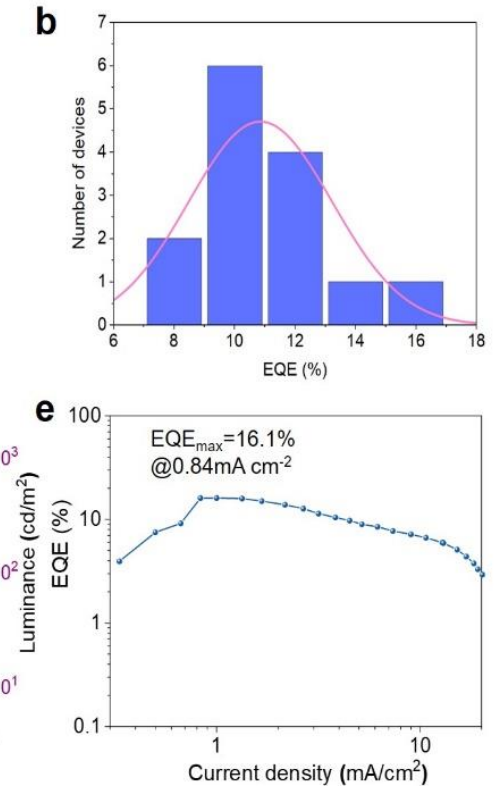

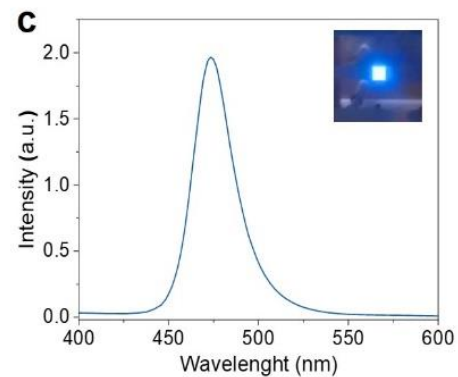

f

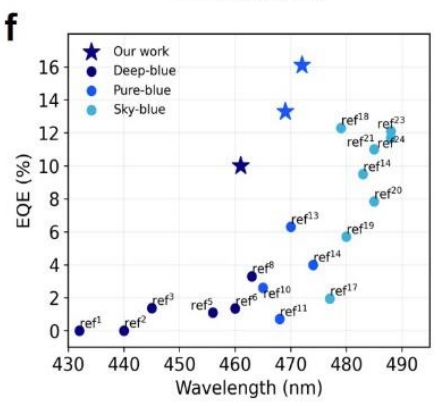

Fig. 4. The performance of blue PeLEDs. a, the schematic diagram shows that charge injection balance can be optimized when the recombination zone is modulated at zone A. $\mathrm{b}$, the histogram of EQEs from 14 devices with the optimized PEDOT:PSS and TPBi thicknesses. c, EL spectrum under forward bias. Inset is a photograph of a working blue PeLED device (device area $\left.\sim 2.25 \mathrm{~mm}^{2}\right)$. d, current density-luminance-voltage $(J-L-V)$ curve, the peak EQE is $16.1 \%\left(0.84 \mathrm{~mA} \mathrm{~cm}^{-2}\right)$. e, EQE- $J$ curve of the champion device. $\mathrm{f}$, comparison of our work with recently reported blue perovskite LEDs with emissions in the range from deep blue to sky blue. 


\section{Supplementary Files}

This is a list of supplementary files associated with this preprint. Click to download.

- SupplementaryinformationHighlyefficientbluelightemittingdiodeswithtunablewavelengthusingverticallygradedbandgapquasi2Dperovskitefilms.pdf 